\title{
The Role of Local Therapy in Multi-focal Epithelioid Haemangioendothelioma
}

\author{
DANIEL TONG ${ }^{1}$, ANASTASIA CONSTANTINIDOU $^{1,2}$, BODIL ENGELMANN $^{1}$, \\ FLORENCE CHAMBERLAIN $^{1}$, KHIN THWAY ${ }^{1,3}$, CYRIL FISHER $^{1,3}$, ANDREW HAYES ${ }^{1}$, \\ NICOS FOTIADIS ${ }^{1}$, CHRISTINA MESSIOU ${ }^{1,3}$, AISHA B. MIAH ${ }^{1,3}$, SHANE H. ZAIDI $^{1}$, \\ CHARLOTTE BENSON ${ }^{1}$, WINETTE VAN DER GRAAF ${ }^{1,3}$ and ROBIN L. JONES ${ }^{1,3}$ \\ ${ }^{1}$ Sarcoma Unit, Royal Marsden Hospital, Fulham, London, U.K.; \\ ${ }^{2}$ Medical School University of Cyprus and BoC Oncology Center, Nicosia, Cyprus; \\ ${ }^{3}$ Institute of Cancer Research, London, U.K.
}

\begin{abstract}
Background/Aim: Epithelioid haemangioendothelioma (EHE) is a rare tumor with a wide spectrum of clinical behavior. There is no consensus on the role of local therapy in symptomatic, multi-focal disease. Patients and Methods: A retrospective review of patients presenting to the Royal Marsden Hospital between January 2000 and December 2017 was conducted. Results: Fifty-three patients with EHE were identified, of which 18 patients (34.0\%) received local therapy, and 11 patients (20.8\%) underwent active surveillance. A variety of local treatment modalities were used with few toxicities, and local recurrence was managed with other local treatments or systemic therapy. Distal disease progression was infrequent $(n=4,7.5 \%)$. Patients who developed pleural effusion $(n=5,9.4 \%)$ had poor outcome irrespective of treatment. Conclusion: Local therapy has a role in a selected patient group managed in a multidisciplinary setting, including patients with indolent disease, and patients with a solitary area of progression/symptomatic disease.
\end{abstract}

Epithelioid haemangioendothelioma (EHE) is a rare vascular tumor originating from endothelial cells (1) with an incidence of one in a million (2). It was first described in 1975 and later termed EHE in 1982 by Weiss and Enzinger (3). It is characterized by the translocation of chromosome 1 and 3 and as a result the fusion of WW domain-containing transcription regulator 1 (WWTR1) and calmodulin-binding

This article is freely accessible online.

Correspondence to: Robin L. Jones, Sarcoma Unit, Royal Marsden Hospital, Fulham Road, London, SW3 6JJ, U.K. Tel: +44 2078082590, Fax: +44 2078082113, e-mail: robin.jones4@nhs.net

Key Words: Epithelioid haemangioendothelioma, local therapy, surgery, radiation, ablation, surveillance, pleural effusion. transcription activator 1 (CAMTA1) (4). It often presents as a multi-focal disease, and can originate in different sites, commonly in the liver and lung. EHE has a wide spectrum of clinical behavior, and can follow an indolent course but can also be very aggressive (1).

Due to its rarity, there are few published data regarding the management of this disease and there is no consensus regarding the optimal management of multi-focal disease. For patients with an indolent disease pattern, active surveillance is considered optimal management. However, there is very little information regarding the safety and efficacy of local therapy in symptomatic or solitary progressive disease. The available literature is limited to case reports documenting the use of a single modality. Therefore, the aim of this study was to analyze the safety and efficacy of local therapy in multi-focal EHE in a series of patients treated at a single referral center.

\section{Patients and Methods}

Local institutional approval was obtained to perform the study. A retrospective search of the prospectively maintained Royal Marsden Sarcoma Unit database was performed to identify EHE patients treated at the Royal Marsden Hospital between January 2000 and December 2017.

The diagnosis of EHE was confirmed in all cases by an experienced soft tissue sarcoma pathologist (KT, CF). Patient demographics, date of diagnosis, disease location, treatment, followup and survival data were obtained from the database and electronic patient record. Patients only seen at the Royal Marsden for a second opinion were not included in this analysis.

Local treatments included were primary resection, re-excision, metastasectomy, radiofrequency ablation (RFA), irreversible electroporation (IRE), liver transplant and radiotherapy (RT). Systemic treatments included chemotherapy, non-steroidal antiinflammatory drug administration such as celecoxib, mTOR inhibition or enrolment in a Phase I trial.

Patients on active surveillance underwent repeat imaging every 3-6 months, with either computed tomography (CT) or magnetic 


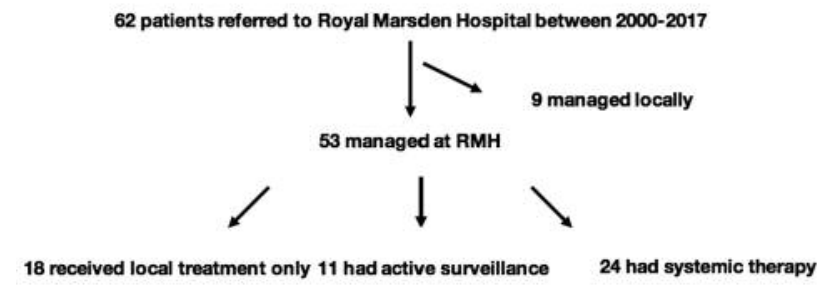

Figure 1. Treatment received by all EHE patients presented to RMH in the period covered by this study.

resonance imaging (MRI). For patients treated with local therapy, response was evaluated 2-3 months following the procedure. Toxicity data were obtained from the electronic patient record.

Descriptive statistics were used. Progression-free survival (PFS) was defined as time between treatment initiation and disease progression. Local disease progression was defined as progression of tumor sites present at staging, and distant progression was progression of distal sites not involved at the time of diagnosis. Overall survival (OS) was defined as time from diagnosis to date of death. Outcomes were presented as median PFS and OS.

\section{Results}

Sixty-two patients with EHE presented to the Royal Marsden Hospital between 2000 and 2017 (Figure 1). Nine patients were excluded from the analysis due to follow-up in local hospitals. Twenty-nine of these patients received local treatments or active surveillance at the Royal Marsden Hospital, while 24 had systemic therapy. The outcomes in this group are reported in a separate publication (5). The median age was 41.5 years (range $=16-72$ ) in the local treatment group and 51 years (range $=29-76$ ) in those under surveillance (Table I). Liver and lung were the most common sites involved. Patient demographics and tumor characteristics at diagnosis are summarized in Table I.

Eighteen patients $(34.0 \%)$ received local treatments for EHE, and 11 patients (20.7\%) had active surveillance (Figure $1)$. Nine $(50 \%)$ of the patients in the local treatment group had multi-focal disease at diagnosis, and all 11 patients in the surveillance group had multi-focal disease (Table I). Two patients in the surveillance group proceeded to have local therapy on disease progression. Primary resection was used in 10 patients, while other first-line treatment modalities in the local treatment group included radiotherapy $(n=6)$, radiofrequency ablation $(\mathrm{n}=1)$, endovascular embolization $(n=1)$, liver transplant $(n=1)$ (Table II). Further lines of local treatments used were metastasectomy $(n=1)$, irreversible electroporation $(n=1)$, and re-excision $(n=2$, with one patient undergoing two further re-excisions following primary surgery). Details of treatment and outcome can be found in Table III.
Table I. Patient demographics and tumor characteristics.

\begin{tabular}{lcc}
\hline & Local treatment & Surveillance \\
\hline & & \\
Number of patients & 18 & 11 \\
Median age & 41.5 & 51 \\
Gender & & \\
Male & 5 & 1 \\
Female & 13 & 10 \\
Multi-focal disease at diagnosis & & \\
Yes & 9 & 11 \\
No & 9 & 0 \\
Sites involved at diagnosis & & \\
Liver & 7 & 7 \\
Lung & 6 & 10 \\
Bone & 4 & 2 \\
Bowel & 1 & 0 \\
Vulva & 2 & 0 \\
Limbs & 3 & 0 \\
Peritoneal & 2 & 0 \\
Mediastinum & 1 & 1 \\
Groin & 1 & 0 \\
Spine & 2 & 0 \\
Lymph node & 1 & 0 \\
\hline
\end{tabular}

Table II. Local treatments given for EHE at RMH.

\begin{tabular}{lc}
\hline Local treatment modality & $\mathrm{n}(\%)$ \\
\hline Surgery & $10(34.5)$ \\
Surgical re-excision & $2(6.9)$ \\
Metastasectomy & $1(3.4)$ \\
Liver transplant & $1(3.4)$ \\
Radiotherapy (including protons) & $6(15.4)$ \\
Radiofrequency ablation & $1(3.4)$ \\
Irreversible electroporation & $1(3.4)$ \\
Endovascular embolization & $1(3.4)$ \\
\hline
\end{tabular}

Surgery +/- other local treatment $(n=10)$. Two (20\%) patients in this group had multi-focal disease at the time of diagnosis. Ten patients had primary resection for EHE, and six did not require further treatment after a median follow-up of 27.5 months. Two patients needed further re-excision of the primary site. The first patient had residual disease excised and was found to have positive regional lymph nodes which were unresectable. The patient then underwent active surveillance, and is well 25 months following the re-excision with no recurrence at the primary site and stable appearance of the nodal disease. The second patient, who had multifocal disease at the time of first surgery, had two further reexcisions of the primary sites following local disease progression at 4 months and 18 months, respectively, from 
the first operation, ultimately resulting in an above-knee amputation. The disease is stable in the lung and liver 6 months following the above knee amputation.

Of the 10 primarily surgically managed patients, eight $(80 \%)$ were free of local recurrence with a median follow-up of 31 months (range=2-56). Local recurrence was found in two patients $(20 \%)$, with a median PFS of 9.5 months. One patient was treated with surgical re-excision as described above and the other with irreversible electroporation (IRE). The other patient had multi-focal disease in the lung and liver and was treated with IRE to the enlarging liver tumors 15 months after primary resection. IRE was performed in two metastases measuring $5.4 \mathrm{~cm}$ and $3.2 \mathrm{~cm}$. The patient is progression-free 9 months following the procedure. Patient No. 2 in Table III had residual disease excised upon review of post-operative pathology and was therefore not deemed to have local recurrence. This patient had residual disease excised and is free of recurrence at the 35-month follow-up.

Nine $(90 \%)$ patients had no evidence of distant recurrence with a median follow-up of 24 months. One patient developed distant recurrence in the liver and underwent metastasectomy 7 months following primary surgery. He is now recurrence-free, 23 months after the procedure.

All 10 patients were alive at the time of analysis, with a median follow-up of 27 months.

In terms of toxicity, one patient developed a seroma postoperatively which was drained, and another patient developed post-operative chronic pain following primary resection of an upper limb EHE requiring ongoing input from the pain team. Other local treatments without primary surgery $(n=8)$. All patients had multi-focal disease at diagnosis. One patient had liver transplantation, six had radiotherapy, one had endovascular embolization followed by proton therapy, and one had radiofrequency ablation. Five patients developed local recurrence (median local $\mathrm{PFS}=8$ months), and two patients received systemic treatment, two underwent surveillance, and one had surgical excision. Three (37.5\%) patients remained disease-free at 21 months median followup. Two patients had distal progression (median distal $\mathrm{PFS}=10.5$ months), one was started on systemic treatment and the other on surveillance. Six (75\%) remained diseasefree at 37 months median follow-up. Four (50\%) patients were alive at the time of analysis (median follow-up= 44.5 months), four had died at the time of analysis and had a median OS of 36.5 months. No significant adverse events were recorded.

One patient had multi-focal disease in the liver and underwent liver transplant one year following diagnosis. She subsequently developed multi-focal liver lesions 31 months post-transplant which slowly increased in size. She eventually had a partial hepatectomy after 18 months of surveillance due to an increase in the size of the lesions, and is recurrence-free at 11 months post-op (72 months follow-up).
One patient underwent endovascular embolization 10 months following diagnosis. The patient then received proton therapy two months following endovascular embolization ( $<18$ years old at diagnosis). The patient developed metastasis one month following completion of proton therapy and received chemotherapy (doxorubicin/ ifosfamide for three cycles, followed by paclitaxel on progression). She passed away five months after starting chemotherapy (OS=21 months).

A patient with disease in the liver, lung, retroperitoneum and bone was treated with RFA to a liver lesion (6 months from diagnosis). There was evidence of local progression in the liver 7 months following RFA. She was started on celecoxib and died 2 months later (OS=15 months).

The two patients described above both developed pleural effusion and the time from event to death was 6 and 2 months respectively.

Radiotherapy was given to six patients for bone metastases. Two received radical treatment one of which was delivered via stereotactic ablative technique and one had proton therapy (outcome described above). The patient with stereotactic ablative radiotherapy had a local PFS of 62 months and on recurrence was given chemotherapy. The patient has stable disease at 11 months follow-up. Among the four patients receiving conventional RT, whilst one did not develop further disease-events (median follow-up 52 months), two patients developed local recurrence (PFS 8 and 9 months respectively) and one developed distant progression at 20 months. These were all managed with active surveillance.

Surveillance group $(n=11)$. Eleven patients with multifocal disease at the time of diagnosis were treated conservatively with active surveillance. Six patients had progression in primary disease sites with a median PFS of 29 months. Of these six, two patients continued on surveillance, one was referred for liver transplant and one for IRE. Two were started on chemotherapy. Three patients in the surveillance group developed pleural effusion. In the surveillance group, there was no progression in five (45.4\%) patients (median follow-up 29 months), and no distal progression in any patient. Six $(54.5 \%)$ patients were alive at the time of analysis (median follow-up 35 months). Five had died at the time of analysis (median OS=29 months), and two of the five deaths were unrelated to malignancy.

Pleural effusion appeared to be a poor prognostic factor in EHE. Among patients treated with local therapy or surveillance, it was present in five $(9.4 \%)$ patients. Three patients had systemic treatment and two were treated conservatively. All five patients had died at time of analysis with a median time from development of pleural effusion to death of five months. 
Table III. Local treatment modalities and their respective outcomes.

\begin{tabular}{|c|c|c|c|c|c|c|c|}
\hline $\begin{array}{l}\text { Patient } \\
\text { no. }\end{array}$ & $\begin{array}{l}\text { Disease } \\
\text { sites }\end{array}$ & $\begin{array}{l}\text { Multi-focal } \\
\text { at diagnosis }\end{array}$ & $\begin{array}{c}\text { Treatment } \\
\text { modality }\end{array}$ & $\begin{array}{l}\text { Interval between } \\
\text { treatment (months) }\end{array}$ & $\begin{array}{c}\text { Local PFS } \\
\text { (months) }\end{array}$ & $\begin{array}{c}\text { Distal PFS } \\
\text { (months) }\end{array}$ & Toxicity \\
\hline \multicolumn{8}{|c|}{ Local treatment surgery } \\
\hline 1 & Lower limb & No & Surgery & 0 & Nil at 56 & Nil at 56 & Nil \\
\hline 2 & Vulva & No & $\begin{array}{l}\text { Surgery \& re-excision } \\
\text { of residual disease }\end{array}$ & 0 & Nil at 35 & Nil at 35 & Nil \\
\hline 3 & Upper limb & No & Surgery & 0 & Nil at 32 & Nil at 32 & Post-op chronic pain \\
\hline 4 & Vulva & No & Surgery & 0 & Nil at 37 & Nil at 37 & Nil \\
\hline \multirow[t]{2}{*}{5} & Bowel, liver & No & Surgery & 0 & Nil at 30 & 7 & Nil \\
\hline & & & Metastasectomy & 7 & Nil at 23 & Nil at 23 & Nil \\
\hline 6 & Peritoneal & No & Surgery & 0 & Nil at 23 & Nil at 23 & Nil \\
\hline \multirow{2}{*}{7} & Liver, lung & Yes & Surgery & 7 & 15 & Nil at 24 & Nil \\
\hline & & & IRE & 15 & Nil at 9 & Nil at 9 & Nil \\
\hline \multirow[t]{3}{*}{8} & Lower limb, lung, liver & Yes & Surgery & 0 & 4 & Nil at 24 & Nil \\
\hline & & & Re-excision & 4 & 14 & Nil at 24 & Nil \\
\hline & & & Re-excision & 14 & Nil at 6 & Nil at 24 & Nil \\
\hline 9 & Groin & No & Surgery & 240 & Nil at 3 & Nil at 3 & Seroma \\
\hline 10 & Liver & No & Surgery & 0 & Nil at 2 & Nil at 2 & Nil \\
\hline
\end{tabular}

Local treatment without surgery

\begin{tabular}{|c|c|c|c|c|c|c|c|}
\hline 11 & Scapula, liver & Yes & Radiotherapy & 4 & Nil at 29 & 20 & Nil \\
\hline \multirow[t]{2}{*}{12} & Liver & Yes & Liver transplant & 12 & 7 & Nil at 60 & Nil \\
\hline & & & Surgery & 49 & Nil at 11 & Nil at 11 & Nil \\
\hline 13 & Bone, lung & Yes & Radiotherapy & 2 & 9 & Nil at 12 & Nil \\
\hline 14 & Mediastinum & Yes & Radiotherapy (SRS) & 11 & 62 & Nil at 73 & Nil \\
\hline 15 & Spine, bone, lung, LN & Yes & Radiotherapy & 37 & Nil at 15 & Nil at 15 & Nil \\
\hline 16 & Bone, lung & Yes & Radiotherapy & 49 & 8 & Nil at 59 & Nil \\
\hline \multirow[t]{2}{*}{17} & Spine & Yes & Endovascular embolization & 3 & Nil at 21 & 5 & Nil \\
\hline & & & Proton therapy & 5 & Nil at 21 & 1 & Nil \\
\hline 18 & $\begin{array}{l}\text { Liver, bone, lung, } \\
\text { retroperitoneum }\end{array}$ & Yes & RFA & 6 & 7 & Nil at 15 & Nil \\
\hline
\end{tabular}

\begin{tabular}{lllll}
\hline Patient no. & Disease sites & Multi-focal at diagnosis & Treatment modality & Local PFS (months)
\end{tabular}

Surveillance

\begin{tabular}{|c|c|c|c|c|c|}
\hline 19 & Liver, lung & Yes & Surveillance & Nil at 26 & Nil at 26 \\
\hline 20 & Liver, lung & Yes & Surveillance & Nil at 106 & Nil at 106 \\
\hline 21 & Liver, lung & Yes & Surveillance & Nil at 158 & Nil at 158 \\
\hline 22 & Liver & Yes & $\begin{array}{l}\text { Surveillance (referred for } \\
\text { liver transplant) }\end{array}$ & 49 & Nil at 51 \\
\hline 23 & Liver, lung & Yes & Surveillance (referred for IRE) & 36 & Nil at 36 \\
\hline 24 & Lung, bone & Yes & Surveillance & 3 & Nil at 24 \\
\hline 25 & Liver, lung & Yes & Surveillance & 39 & Nil at 46 \\
\hline 26 & Lung & Yes & Surveillance & 2 & Nil at 73 \\
\hline 27 & Liver, lung & Yes & Surveillance & 22 & Nil at 34 \\
\hline 28 & Lung, mediastinum & Yes & Surveillance & Nil at 29 & Nil at 29 \\
\hline 29 & Lung, bone & Yes & Surveillance & Nil at 2 & Nil at 2 \\
\hline
\end{tabular}

\section{Discussion}

EHE is a rare disease with a wide spectrum of clinical behavior ranging from very indolent to an extremely aggressive life-threatening disease. There are limited published data regarding the use of local therapy in multi-focal EHE.
Our study suggests that local therapy may have a role even in patients with multi-focal disease in selected patients managed within a multidisciplinary team. Our study is of course limited by its retrospective nature and relatively small patient population. However, it does provide a benchmark for the use of local therapy in multi-focal EHE. Due to the often-indolent 
nature of the disease, resection of symptomatic/ progressing lesions could be considered. The pros and cons of this approach should be considered with each individual patient. In our series, $80 \%$ of our patients having upfront primary resection did not have multi-focal disease at the time of diagnosis. Our experience is that surgical excision improves local PFS compared to active surveillance when disease burden is assessed, but distal PFS is similar.

A number of retrospective studies have reported the outcome of EHE patients treated with liver transplantation (LT) (6-8). These studies are limited by an inherent selection bias and their retrospective nature. Given that many patients with multi-focal EHE have indolent disease and are on active surveillance, it is difficult to define precisely the role of liver transplantation in this disease, i.e. patients treated with transplantation may have indolent disease and it is possible that they would have a good outcome without transplantation. Bonaccorsi-Riani et al. suggested that EHE patients receiving LT have a 5-year overall survival of $83 \%$ and progression-free survival of $82 \%$, based on the results from the European Liver Intestinal Transplant Association, European Liver Transplant Registry (ELTR) (6-7). However, it is unclear whether LT influenced outcome in these patients, as EHE can often be very indolent. Grotz et al. conducted a retrospective review of 30 patients treated in a single center. Five-year OS was worse $(\mathrm{p}<0.05)$ in those presenting with liver-only disease with diffuse disease pattern (disease involving $>4$ lobes, $>10$ tumor nodules, tumor size $>10 \mathrm{~cm})(8)$. LT was recommended in those presenting with these risk factors. Lai et al. analyzed data from the ELTR and proposed a risk score (HEHE-LT) to stratify patients to identify those who would benefit from LT according to risk of recurrence after transplantation (9). The risk factors associated with recurrence that were identified include pathological invasion of hilar lymph nodes and macrovasculature, and, interestingly, a short waiting time to transplantation $(<120$ days $)$. This suggests that an assessment of the pace of disease progression is crucial before planning extensive surgical procedures.

Radiotherapy (RT) is normally used in EHE as an adjunct to other treatments such as chemotherapy. Current literature on the role for radiotherapy in EHE are mostly described in single case reports. For example, Albakr et al. described a 34-year-old with thoracic spinal EHE treated with adjuvant radiotherapy following surgical decompression for an epidural tumor causing cord compression (10). Similarly, Guy et al. published a case of a vertebral EHE receiving volumetric intensity-modulated radiotherapy following failure of surgery (11). A number of case series have investigated the role of RT in EHE with bone involvement. Wold et al. presented 38 patients with bony EHE treated with RT (12). In 21 patients RT was used as monotherapy, 8 in adjuvant setting, and 9 combined with chemotherapy. $48 \%$ of these achieved local control, and $93 \%$ of these were in the group with the higher radiotherapy dose (>50 Gy). At median follow-up of 2.9 years, OS was $75 \%$ in the higherdose group and $40 \%$ for those who received a lower dose. Yim et al. suggested that RT may be used in cases where radical resection is not possible, although the evidence of this is not clear (13).

There are limited data regarding radiation in this disease, but it can be used for palliation of symptomatic lesions.

Radiofrequency ablation (RFA) is commonly used to treat hepatic lesions. The use of RFA in bony EHE has been described in case reports. Davis et al. treated multi-focal EHE with bony lesions with wide excision of soft tissue lesions and RFA to bony lesions (14). Rosenthal et al. reported the use of RFA when primary resection alone would result in significant co-morbidity due to the location of tumor (15). RFA was recommended as an adjunct to limit the extent of surgery, particularly when it would result in significant cosmetic or functional deficit.

Irreversible electroporation (IRE) is a non-thermal focal ablation technique and its use in hepatic EHE has been described in a case report, documenting significant reduction in FDG-PET uptake following IRE (16). IRE was used in this particular case because RFA was contraindicated due to the vasculocentric nature of the target lesions. RFA in such lesions with low vascularity would likely not sustain a therapeutic response (16). IRE is a therapeutic modality warranting further exploration in EHE.

In summary, EHE is a rare condition, and is best managed at referral centers with a multidisciplinary approach. Local therapy can be considered for patients with indolent disease, including those with indolent multifocal disease with a solitary area of progression/symptomatic lesion. Pleural effusion is a poor prognostic marker in EHE, and early involvement of the palliative care team is particularly important in this group.

\section{Conflicts of Interest}

RL Jones has received honoraria and consultation fees from Adaptimmune, Blueprint, Clinigen, Eisai, Epizyme, Daichii, Deciphera, Immunedesign, Lilly, Merck, Pharmamar and Upto Date.

\section{Acknowledgements}

Clinical nurse specialists: Alison Dunlop, Kelly McKibbin, Karen O'Meara, Angela Teague. Biomedical Research Center at The Royal Marsden and The Institute of Cancer Research.

\section{Authors' Contributions}

DT- Designed the study, collected and analyzed data, wrote the manuscript; AC- Analyzed data, edited the manuscript; BECollected the data, edited the manuscript; FC, KT, CF, AH, NF, CM, AM, SZ, CB, WG- Edited the manuscript; RJ- Conceived and designed the study, edited the manuscript. 


\section{References}

1 Sardaro A, Bardoscia L, Petruzzelli MF and Portaluri M: Epithelioid hemangioendothelioma: an overview and update on a rare vascular tumor. Oncol Rev 8(2): 82-91, 2014. PMID: 25992243. DOI: $10.4081 /$ oncol.2014.259

2 Mehrabi A, Kashfi A, Fonouni H, Schemmer P, Schmied BM, Hallscheidt P, Schirmacher P, Weitz J, Friess H, Buchler MW and Schmidt J: Primary malignant hepatic epithelioid hemangioendothelioma: a comprehensive review of the literature with emphasis on the surgical therapy. Cancer 107(9): 21082121, 2006. PMID: 17019735. DOI: 10.1002/cncr.22225

3 Weiss S and Enzinger FM: Epithelioid hemangioendothelioma: a vascular tumor often mistaken for a carcinoma. Cancer 50(5): 970-981, 1982. PMID: 7093931. DOI: 10.1002/1097-0142 (19820901)50:5<970::aid-cncr2820500527>3.0.co;2-z

4 Tanas M, Tanas MR, Sboner A, Oliveira AM, Erickson-Johnson MR, Hespelt J, Hanwright PJ, Flanagan J, Luo Y, Fenwick K, Natrajan R, Mitsopoulos C, Zvelebil M, Hoch BL, Weiss SW, Debiec-Rychter M, Sciot R, West RB, Lazar AJ, Ashworth A, Reis-Filho JS, Lord CJ, Gerstein MB, Rubin MA and Rubin BP: Identification of a disease-defining gene fusion in epithelioid hemangioendothelioma. Sci Transl Med 3(98): 98ra82, 2011. PMID: 21885404. DOI: 10.1126/scitranslmed .3002409

5 Yousaf N, Maruzzo M, Judson I, Al-Muderis O, Fisher C and Benson C: Systemic treatment options for epithelioid haemangioendothelioma: The Royal Marsden Hospital experience. Anticancer Res 35(1): 473-480, 2015. PMID: 25550590.

6 Bonaccorsi-Riani E and Lerut JP: Liver transplantation and vascular tumours. Transpl Int 23(7): 686-691, 2010. PMID: 20492619. DOI: 10.1111/j.1432-2277.2010.01107.x

7 Lerut JP, Orlando G, Adam R, Schiavo M, Klempnauer J, Mirza D, Boleslawski E, Burroughs A, Sellés CF, Jaeck D, Pfitzmann R, Salizzoni M, Söderdahl G, Steininger R, Wettergren A, Mazzaferro V, Le Treut YP, Karam V and European Liver Transplant Registry: The place of liver transplantation in the treatment of hepatic epithelioid hemangioendothelioma: Report of the European liver transplant registry. Ann Surg 246(6): 949-957, 2007. PMID: 18043096. DOI: 10.1097/SLA.0b013e3 1815c2a70

8 Grotz TE, Nagorney D, Donohue J, Que F, Kendrick M, Farnell M, Harmsen S, Mulligan D, Nguyen J, Rosen C and ReidLombardo KM: Hepatic epithelioid haemangioendothelioma: Is transplantation the only treatment option? HPB (Oxford) 12(8): 546-553, 2010. PMID: 20887322. DOI: 10.1111/j.1477-2574. 2010.00213.x
9 Lai Q, Feys E, Karam V, Adam R, Klempnauer J, Oliverius M, Mazzaferro V, Pascher A, Remiszewski P, Isoniemi H, Pirenne J, Foss A, Ericzon BG, Markovic S, Lerut JP and European Liver Intestine Transplant Association: Hepatic epithelioid hemangioendothelioma and adult liver transplantation: Proposal for a prognostic score based on the analysis of the ELTR-ELITA registry. Transplantation 101(3): 555-564, 2017. PMID: 28212256. DOI: $10.1097 /$ TP.0000000000001603

10 Albakr A, Schell M, Drew B and Cenic A: Epithelioid hemangioendothelioma of the spine: case report and review of the literature. J Spine Surg 3(2): 250-259, 2017. PMID: 2874 4509. DOI: 10.21037 jss.2017.05.05

11 Guy JB, Trone JC, Chargari C, Falk AT, Khodri M and Magné $\mathrm{N}$ : Epithelioid hemangioendothelioma of the spine treated with RapidArc volumetric-modulated radiotherapy. Med Dosim 39(3): 242-245, 2014. PMID: 24833300. DOI: 10.1016/ j.meddos.2014.03.002

12 Wold L, Unni KK, Beabout JW, Ivins JC, Bruckman JE and Dahlin DC: Hemangioendothelial sarcoma of bone. Am J Surg Pathol 6(1): 59-70, 1982. PMID: 7200731.

13 Yim KL, Sumathi VP and Spooner D: Radiotherapy as an effective primary treatment for epithelioid haemangioendothelioma of the cervical spine. Anticancer Res 32(10): 4597-4600, 2012. PMID: 23060592.

14 Davis AT, Guo AM, Phillips NJ and Greenberg DD: A novel treatment for bone lesions of multi-focal epithelioid sarcomalike hemangioendothelioma. Skeletal Radiol 44(7): 1013-1019, 2015. PMID: 25564226. DOI: 10.1007/s00256-014-2089-x

15 Rosenthal DI, Treat ME, Mankin HJ, Rosenberg AE and Jennings CL: Treatment of epithelioid hemangioendothelioma of bone using a novel combined approach. Skeletal Radiol 30(4): 219-222, 2001. PMID: 11392296.

16 Neal RE, Kavnoudias H, Cheung W, Golebiowski B, McLean CA and Thomson KR: Hepatic epithelioid hemangioendothelioma treated with irreversible electroporation and antibiotics. J Clin Oncol 31(27): e422-e426, 2013. PMID: 23897957. DOI: $10.1200 / J C O .2012 .44 .9736$ 